\title{
Original
}

\section{Comparison of two modified methods of intrathecal catheterization in rats}

\author{
Ganggang $\mathrm{KONG}^{1,2)}$, Zhiping $\mathrm{HUANG}^{2)}$, Qingan $\mathrm{ZHU}^{2)}$ and Yong WAN ${ }^{1)}$ \\ 1) Department of Spine Surgery, The First Affiliated Hospital, Sun Yat-sen University, No. 58, Zhong Shan Er Lu, Guangzhou \\ 510080, Guangdong, China \\ 2)Department of Spinal Surgery, Nanfang Hospital, Southern Medical University, 1838 N. Guangzhou Ave., Guangzhou, \\ 510515, China
}

\begin{abstract}
The study designed to compare two different methods of intrathecal catheterization in rats and to develop a simple and safe drug administration in cervical spinal canal of rats. The subarachnoid catheterization was performed via either atlanto-occipital membrane or laminectomy at L3-4 in rats. Body weight, Basso, Beattie, and Bresnahan (BBB) locomotion rating scores and forelimb locomotor rating scale (FLS) were measured on preoperative day 1 and postoperative day $1,7,14$, respectively. FLS score of $37.5 \%$ rats and BBB score of $50 \%$ rats in the atlanto-occipital approach (AOA) group decreased, but no rats showed locomotor impairment in the lumber approach (LA) group. The mean body weight of rats in AOA group reduced significantly compared with LA group. In LA group, $62.5 \%$ of catheter tips were located at T1, and in AOA group, the tips of catheter located at $\mathrm{C} 2$ in $62.5 \%$ cases. The PE10 catheter can be successfully inserted into the spinal intrathecal space for chronic delivery of drugs either via L3-L4 interlaminar space or via atlanto-occipital membrane. And the subarachnoid catheterization via L3-L4 interlaminar space could be easily placed at T1 with little complication.

Key words: atlanto-occipital membrane, intrathecal catheterization, spinal cord injury
\end{abstract}

\section{Introduction}

Subarachnoid catheterization was an important method for neurobiology research. There are two main techniques for subarachnoid catheterization, that is, atlantooccipital membrane approach and lumbar laminectomy approach. However, several defects of the techniques were still remained, including neurological defects, postoperative mortality and subarachnoid hemorrhage $[2,6]$.

We previously developed a new C5 unilateral spinal cord injury model in rats $[3,4]$. For chronic in-situ drug delivery, we currently modified the classical techniques and compared the safety and effectiveness of catheterization through atlanto-occipital membrane or lumber laminectomy at L3-4.

\section{Materials and Methods}

\section{Experimental animals}

This work was approved by the Animal Care and Use Committee of Sun Yat-sen University. Adult male Sprague Dawley (SD) rat weighing 275-325 g were used for the animal experiments. Rats were provided food and water ad libitum with a $12: 12 \mathrm{~h}$-light cycle at $22-26^{\circ} \mathrm{C}$. The animals were randomly divided into 2 groups, the lumber approach (LA) group and the atlanto-occipital approach (AOA) group.

Subarachnoid catheterization via lumber approach

After anesthesia with $2.0 \%$ isoflurane in $\mathrm{O}_{2}$, rats in LA group were fixated on the stereotaxic apparatus in prone position. An incision above L3-L4 interlaminar space was made, and then the paravertebral muscles were

(Received 24 August 2019 / Accepted 2 December 2019 / Published online in J-STAGE 20 December 2019)

Corresponding author: Y. Wan.e-mail: yongwan65@163.com

This is an open-access article distributed under the terms of the Creative Commons Attribution Non-Commercial No Derivatives (by-nc-nd) License <http://creativecommons.org/licenses/by-nc-nd/4.0/>.

(C)2020 Japanese Association for Laboratory Animal Science 

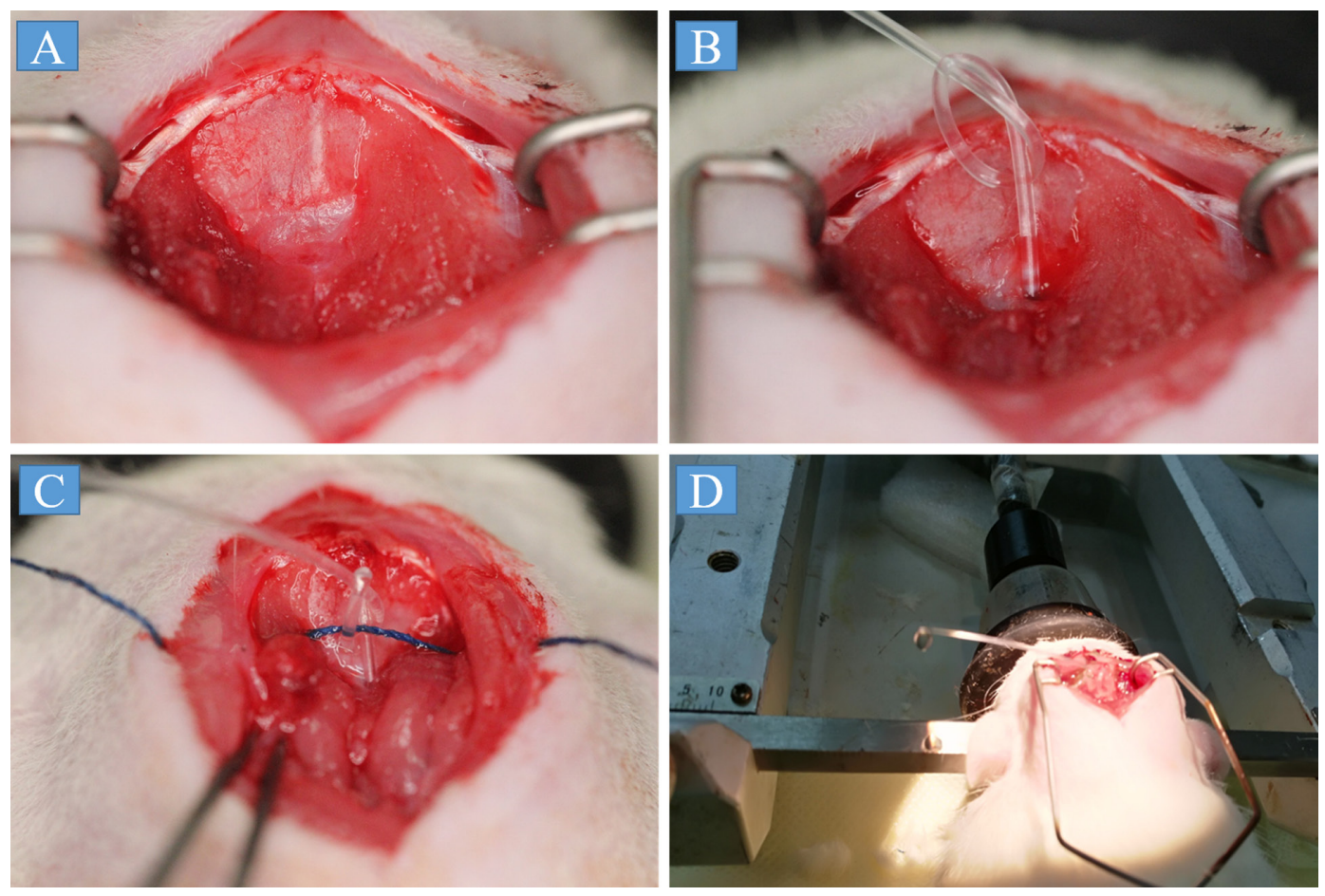

Fig. 1. Illustration of the procedures of subarachnoid catheterization via atlanto-occipital membrane. A: Expose the atlanto-occipital membrane. B: The catheter was inserted advanced caudally and parallel with the dorsal surface of the brainstem. C: The catheter was fixed with a suture line. D: The backflow of spinal fluid was seen at the catheter orifice.

separated to expose the interlaminar space. After lumbar puncture was performed using a 26-gauge syringe needle through L3-L4 interlaminar space, a sterile PE10 catheter was inserted, a tail-flick was used as the sign of correct position. The catheter was then advanced cephalically to $\mathrm{C} 5$ through the puncture hole. The correct intrathecal localization of the catheter was confirmed by backflow of spinal fluid. The catheter was fixed, and the incision was subsequently sutured.

\section{Subarachnoid catheterization via atlanto-occipital approach}

We modified the procedure of subarachnoid catheterization via atlanto-occipital membrane in rats described by Yaksh and Rudy [11]. Briefly, rats in AOA group were laid on the stereotaxic apparatus in prone position after anesthetized. An $1 \mathrm{~cm}$ longitudinal incision was made over the posterior cranio-cervical junction. Muscles were bluntly separated to expose the atlanto-occipital membrane, than the tip of a 26-gauge syringe needle was used to make a hole on the membrane. After measurement the distance between the hole and spinous process of $\mathrm{C} 5$, an appropriate sterile saline-filled PE10 catheter was implanted smoothly into the subarachnoid space of cervical spine parallel with the dorsal surface of the brainstem. Backflow of spinal fluid could demonstrated the correct intrathecal localization of the catheter (Fig. 1). The wound was sutured layer by layer, and penicillin was intramuscular injected on the bilateral hind limb. After the surgery, animals were housed in individual cages for recovery.

\section{Drug administration test}

The sterile saline solution was used for drug administration test. For a single injection, $40 \mu 1$ saline solution was administration through the inserted catheter by connecting with a micro-syringe in both groups (Fig. 2).

\section{Neurological impairment}

Deficits of behavior after SCI were scored according to Basso, Beattie, and Bresnahan (BBB) locomotion rating scale, which scored from 0 to 21 as described previously [1]. The hind limb movements, body weight support, forelimb to hind limb coordination, and whole body movements were assessed in the scale. Two experienced researches, blinded to experimental treatment, evaluated open-field locomotion of rats after SCI.

Forelimb locomotor rating scale (FLS) was used as described previously [9]. The scale assessed the forelimbs movement. The scores indicate forelimb joint movements, weight supported stepping, and distal motor control involving paw placement and toe clearance.

Body weight was measured at different time points post-surgery. The animals were sacrificed 14 days after 

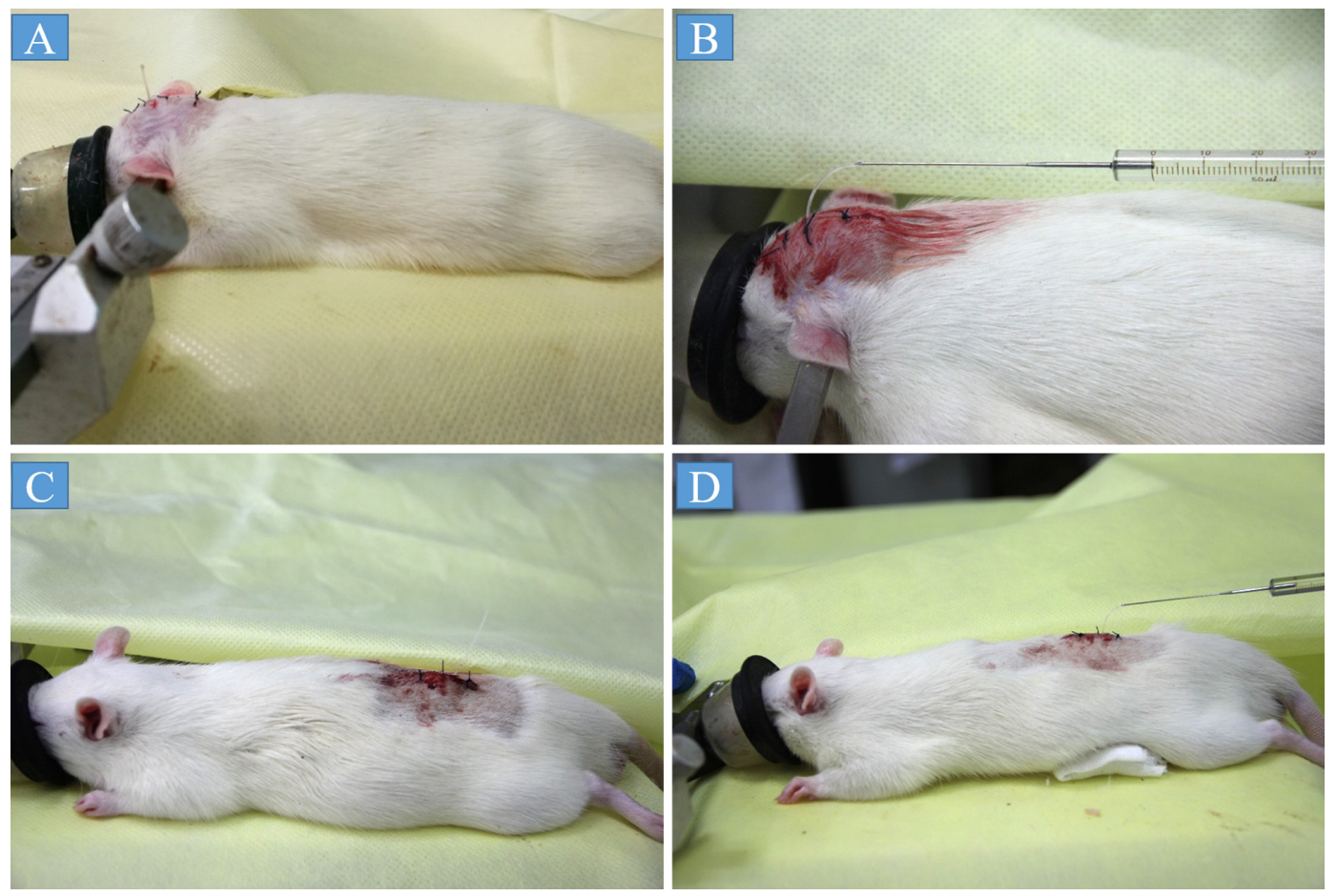

Fig. 2. Illustration of drug administration through the inserted catheter. A: Subarachnoid catheterization via atlanto-occipital membrane. B: The drug solution was administration through the inserted catheter by connecting with a micro-syringe in the atlanto-occipital approach (AOA) group. C: Subarachnoid catheterization via L3-L4 interlaminar space. D: The drug solution was administration through the inserted catheter by connecting with a micro-syringe in the lumber approach (LA) group.
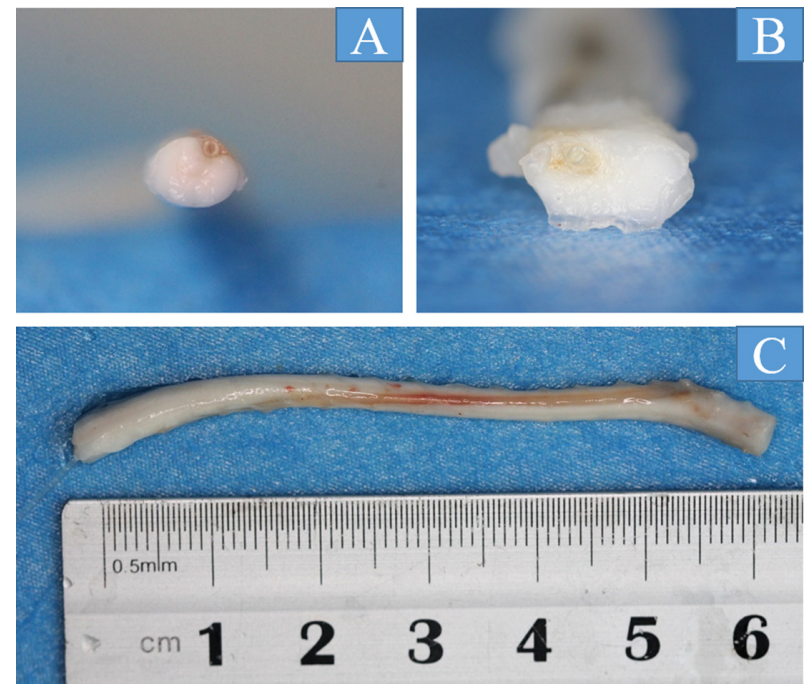

Fig. 3. Illustration of the position of inserted catheter in subarachnoid. A and C: Axial and sagittal view of the catheter in the lumber approach (LA) group, the tip of catheter located totally in subarachnoid. B: Axial view of the catheter tip in the atlanto-occipital approach (AOA) group. The invasion of the spinal cord by the tip of catheter was seen.

the operation. The length of inserted catheter was measured by a standard measuring ruler, and the position of inserted catheter was marked by the vertebral level (Fig. $3)$.

\section{Data analysis}

The data were presented as means \pm SEM. Statistical differences between various groups were analyzed by two-way analysis of variance (ANOVA) using GraphPad Prism 5 (La Jolla, CA, USA), or Student's $t$-test using SPSS 20.0 (IBM, Armonk, NY, USA) software.

\section{Results}

\section{General data}

There were 8 rats in each group. The body weight in LA group was significantly higher than in AOA group 7 and 14 days after the surgery (Fig. 4).

\section{Neurological impairment}

After the operation, FLS score of $37.5 \%$ rats and BBB score of $50 \%$ rats in AOA group decreased, but no rats showed locomotor impairment in LA group. The lowest FLS and BBB scores showed 3 days after the surgery. And FLS score in AOA group was significantly lower than in LA group 3 days post-surgery (Fig. 5).

\section{Measurements of inserted catheters}

The length of inserted catheter was $7.32 \pm 0.53 \mathrm{~cm}$ in LA group, and $1.05 \pm 0.23 \mathrm{~cm}$ in AOA group (Fig. 6).

The positions of the inserted catheter tips were re- 


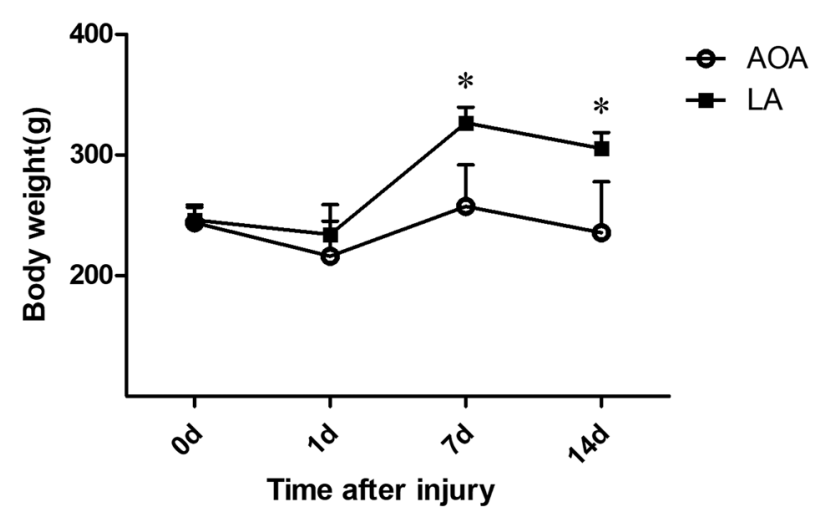

Fig. 4. Body weight was measured at different times. The body weight in the lumber approach (LA) group was significantly higher than in the atlanto-occipital approach (AOA) group 7 and 14 days after the surgery. Data represent mean $\pm \mathrm{SD}$ of at least three independent experiments $(\mathrm{n}=8$ per group). ${ }^{*} P<0.05$ versus the AOA group.

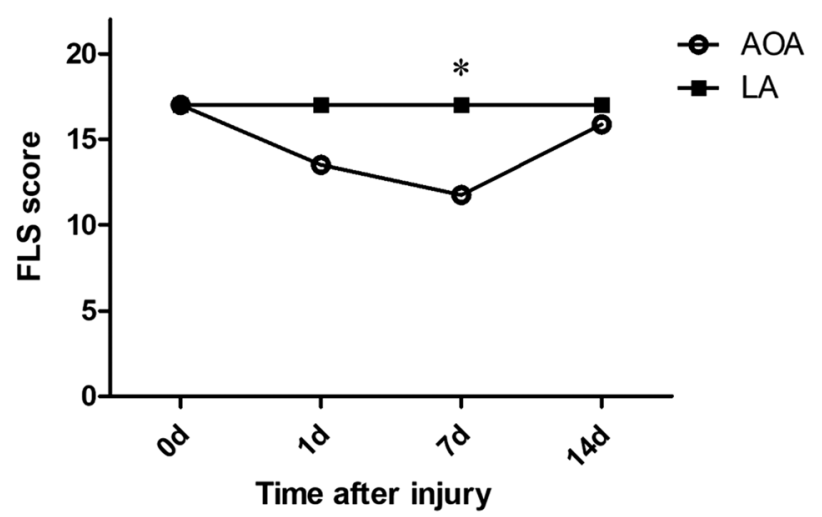

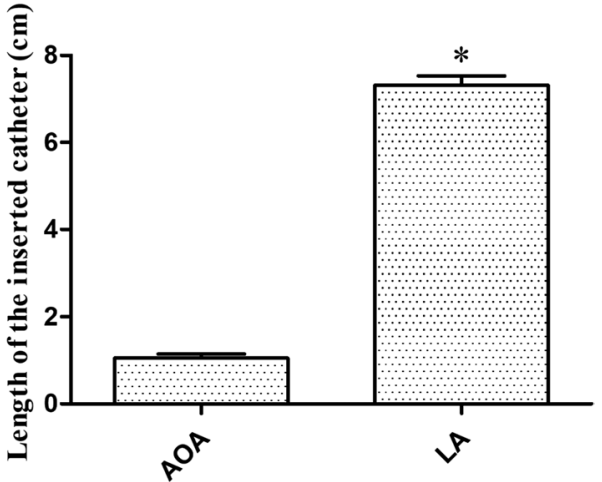

Fig. 6. The length of inserted catheter was measured. The mean length of inserted catheter was significantly longer than in the atlanto-occipital approach (AOA) group. Data represent mean \pm $\mathrm{SD}$ of at least three independent experiments ( $\mathrm{n}=8$ per group). ${ }^{*} P<0.05$ versus the AOA group.

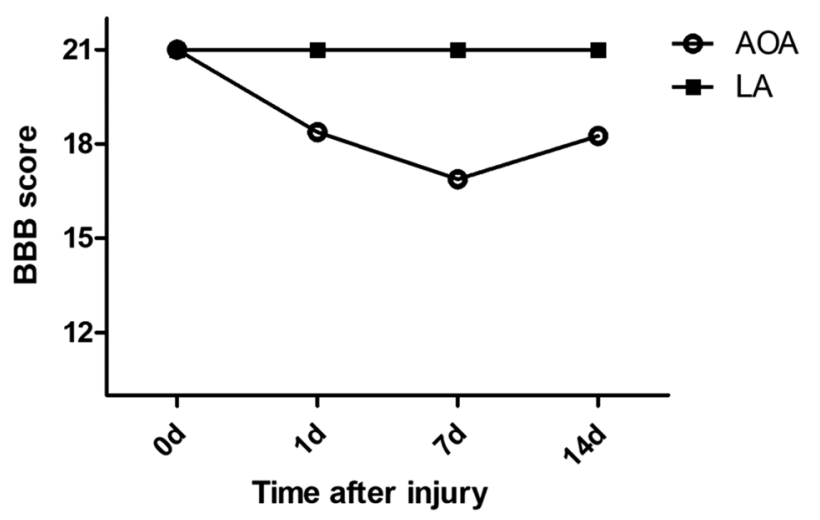

Fig. 5. Neurological defects was evaluated using forelimb locomotor rating scale (FLS) and Basso, Beattie, and Bresnahan (BBB) scores. After the catheterization, FLS and BBB scores in the atlanto-occipital approach (AOA) group reduced during the first week, than increased. No FLS and BBB scores changed in the lumber approach (LA) group. Significant difference of FLS score between the two group was found at $7 \mathrm{~d}$ after the operation. Data represent mean of at least three independent experiments $(n=8$ per group). ${ }^{*} P<0.05$ versus the AOA group.

Table 1. The position of catheter tip in rats

\begin{tabular}{lcccccccc}
\hline Case & 1 & 2 & 3 & 4 & 5 & 6 & 7 & 8 \\
\hline AOA & C2 & C1 & C2 & C2 & C3 & C3 & C2 & C2 \\
LA & T 1 & T 1 & T2 & C7 & T 1 & T 1 & C7 & T1 \\
\hline
\end{tabular}

corded in Table 1. In AOA group, the inserted catheter tips located in the 2 nd cervical vertebra plane were found in 5 cases, 2 cases in the 3rd cervical vertebra plane, and 1 case in the 1 st cervical vertebra plane. In LA group, the inserted catheter tips located in the 1 st thoracic vertebra plane were found in 5 cases, 2 cases in the 7 th cervical vertebra plane, and 1 case in the 2 nd thoracic vertebra plane.

\section{Discussion}

Subarachnoid catheterization is very important for animal studies for continuous subarachnoid drug administration, especially in spinal cord injury and pain researches. Classical methods including intrathecal catheterization via atlanto-occipital membrane, lumbar laminectomy and thoracic laminectomy were described previously. Although these methods have been used for many years,some limitations were still remain, such as spinal cord injury and high mortality. Previous studies showed that the mean body weight was reduced during the first week, $10-30 \%$ of the animals had varying degrees of neurological impairment, and 3-5\% of the animals died during the initial few days after atlanto-occipital catheterization $[5,8]$. Størkson RV et al. compared the intrathecal catheterization through atlanto-occipital 
membrane and lumbar laminectomy, the lumbar catheterization performed in their study was inserted from L5/ L6 interlaminar space to T12, atlanto-occipital approach was from atlanto-occipital membrane to lumbar enlargement, results indicated that atlanto-occipital catheterization had higher mortality and the rate of neurological symptoms, which was similar to our current study [10]. Mazur $\mathrm{C}$ et al. modified the traditional method of lumbar laminectomy approach, and results showed their method also minimized spinal cord compression with the entire catheter resided in the cauda equina space compared with atlanto-occipital approach [7].

We recently developed a C5 cervical spinal cord injury model in rats [3]. For continuous subarachnoid in situ drug administration, we modified the subarachnoid catheterization either via L3-L4 interlaminar space or via atlanto-occipital membrane. After lumber or atlantooccipital membrane puncture, the catheter was inserted carefully toward C5. The tip of catheter could not placed exactly at $\mathrm{C} 5$, in LA group, it was usually stuck at the cervicothoracic junction, and finally located at $\mathrm{T} 1 \mathrm{in}$ $62.5 \%$ cases. And in AOA group, the tips of catheters located at $\mathrm{C} 2$ in $62.5 \%$ cases.

From the body weight, FLS and BBB scores, the current study demonstrated higher rate of neurological defects and lower mean body weight in AOA group than in LA group, which was similar with previous results [10]. It maybe because the anatomy of posterior craniocervical junction had a large anterior convex angle, brain stem and spinal cord in this region were very fragile. And in LA group, the catheter was inserted from the lumbar cistern with only cauda equina nerves existence, it was safe for avoiding spinal cord injury.

The PE10 catheter can be successfully inserted into the spinal intrathecal space for chronic delivery of drugs either via L3-L4 interlaminar space or via atlanto-occipital membrane. And for cervical spinal cord in situ drug administration, the subarachnoid catheterization via L3-L4 interlaminar space was recommended, because the catheter could be easily placed at $\mathrm{T} 1$ with little complication.
Conflict of Interests

All authors claim that there are no conflicts of interest.

\section{Acknowledgments}

This study was supported by grants from China Postdoctoral Science Foundation (2018M643328).

\section{References}

1. Basso, D.M., Beattie, M.S. and Bresnahan, J.C. 1995. A sensitive and reliable locomotor rating scale for open field testing in rats. J. Neurotrauma 12: 1-21. [Medline] [CrossRef]

2. Hou, Y., Wang, L., Gao, J., Jin, X., Ji, F. and Yang, J. 2016. A modified procedure for lumbar intrathecal catheterization in rats. Neurol. Res. 38: 725-732. [Medline] [CrossRef]

3. Huang, Z., Li, R., Liu, J., Huang, Z., Hu, Y., Wu, X. and Zhu, Q. 2018. Longitudinal electrophysiological changes after cervical hemi-contusion spinal cord injury in rats. Neurosci. Lett. 664: 116-122. [Medline] [CrossRef]

4. Kong, G., Huang, Z., Ji, W., Wang, X., Liu, J., Wu, X., Huang, Z., Li, R. and Zhu, Q. 2017. The Ketone Metabolite $\beta$-Hydroxybutyrate Attenuates Oxidative Stress in Spinal Cord Injury by Suppression of Class I Histone Deacetylases. J. Neurotrauma 34: 2645-2655. [Medline] [CrossRef]

5. Kristensen, J.D., Post, C., Gordh, T. Jr. and Svensson, B.A. 1993. Spinal cord morphology and antinociception after chronic intrathecal administration of excitatory amino acid antagonists in the rat. Pain 54: 309-316. [Medline] [CrossRef]

6. Martin, H., Kocher, L. and Chery-Croze, S. 1984. Chronic lumbar intrathecal catheterization in the rat with reducedlength spinal compression. Physiol. Behav. 33: 159-161. [Medline] [CrossRef]

7. Mazur, C., Fitzsimmons, B., Kamme, F., Nichols, B., Powers, B. and Wancewicz, E. 2017. Development of a simple, rapid, and robust intrathecal catheterization method in the rat. J. Neurosci. Methods 280: 36-46. [Medline] [CrossRef]

8. Schoeffler, P., Auroy, P., Bazin, J.E., Taxi, J. and Woda, A. 1991. Subarachnoid midazolam: histologic study in rats and report of its effect on chronic pain in humans. Reg. Anesth. 16: 329-332. [Medline]

9. Singh, A., Krisa, L., Frederick, K.L., Sandrow-Feinberg, H., Balasubramanian, S., Stackhouse, S.K., Murray, M. and Shumsky, J.S. 2014. Forelimb locomotor rating scale for behavioral assessment of recovery after unilateral cervical spinal cord injury in rats. J. Neurosci. Methods 226: 124-131. [Medline] [CrossRef]

10. Størkson, R.V., Kjørsvik, A., Tjølsen, A. and Hole, K. 1996. Lumbar catheterization of the spinal subarachnoid space in the rat. J. Neurosci. Methods 65: 167-172. [Medline] [CrossRef]

11. Yaksh, T.L., and Rudy, T.A. 1976. Chronic catheterization of the spinal subarachnoid space. Physiol. Behav. 17: 10311036. [Medline] [CrossRef] 